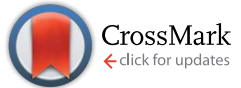

Cite this: Chem. Sci., 2016, 7, 5523

Received 22nd March 2016 Accepted 7th May 2016

DOI: $10.1039 / \mathrm{c} 6 \mathrm{sc} 01279 \mathrm{e}$

www.rsc.org/chemicalscience

\section{Mechanistic elucidation guided by covalent inhibitors for the development of anti-diabetic PPAR $\gamma$ ligands $\uparrow$}

\author{
Hwan Bae, ${ }^{a}$ Jun Young Jang, ${ }^{a}$ Sun-Sil Choi, ${ }^{b}$ Jae-Jin Lee, ${ }^{c}$ Heejun Kim, ${ }^{a}$ Ala Jo, ${ }^{a}$

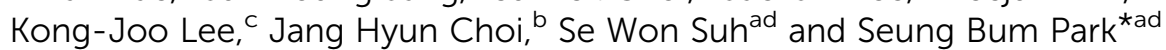

\begin{abstract}
Peroxisome proliferator-activated receptor gamma (PPAR $\gamma$ ) is a ligand-regulated transcription factor that plays crucial roles in adipogenesis, lipid metabolism, and glucose homeostasis. Several PPAR $\gamma$ ligands possess anti-diabetic activity and they commonly inhibit the phosphorylation of PPAR $\gamma$ at serine 273 (Ser273). The recently reported PPAR $\gamma$ ligand SR1664, which selectively blocks the phosphorylation of PPAR $\gamma$ without classical agonism, has potent anti-diabetic activity, indicating that the inhibition of Ser273 phosphorylation is sufficient to provoke anti-diabetic effects. In this study, we revealed the $\mathrm{X}$-ray structure of PPAR $\gamma$ co-crystallized with SR1664 bound to the alternate binding site of PPAR $\gamma$ and confirmed that the alternate site binding of SR1664 blocks the phosphorylation of Ser273. Furthermore, using covalent inhibitors as chemical tools, we demonstrated that the inhibition of phosphorylation is attributed to the occupation of a specific site which is a hydrophobic region between helix 3 and $\beta 3-\beta 4$ at the binding pocket of PPAR $\gamma$. In high-fat diet-induced obese mice, we confirmed the anti-diabetic activity of our covalent inhibitor SB1453 that was designed to bind at the specific site in PPAR $\gamma$ for blocking the phosphorylation of Ser273. Lastly, the target selectivity of SB1453 was demonstrated by fluorescence-based visualization of target proteins complexed with the covalent probe 11 containing a bioorthogonal functional group.
\end{abstract}

\section{Introduction}

Insulin resistance, a major symptom of type II diabetes, is a condition in which body cells become resistant to the normal actions of insulin. ${ }^{1}$ Because of the potent insulin-sensitizing effects of peroxisome proliferator-activated receptor gamma $(\operatorname{PPAR} \gamma)$-targeting drugs such as rosiglitazone and pioglitazone, PPAR $\gamma$ has been considered to be a major therapeutic target for the treatment of type II diabetes, although the underlying molecular mechanisms are still unclear. ${ }^{2-4}$ However, Choi et al. proposed a plausible mechanism by which anti-diabetic PPAR $\gamma$ ligands affect insulin sensitivity. ${ }^{5}$ They demonstrated that the obesity-induced phosphorylation of PPAR $\gamma$ at serine 273 (Ser273) results in the dysregulation of a subset of genes

${ }^{a}$ Department of Chemistry, Seoul National University, Seoul 151-747, Korea. E-mail: sbpark@snu.ac.kr; Fax: +82 28844025

${ }^{b}$ Department of Biological Science, Ulsan National Institute of Science and Technology, Ulsan 689-798, Korea

${ }^{c}$ Graduate School of Pharmaceutical Sciences and College of Pharmacy, Ewha Womans University, Seoul 120-750, Korea

${ }^{d}$ Department of Biophysics and Chemical Biology, Seoul National University, Seoul 151-747, Korea

$\dagger$ Electronic supplementary information (ESI) available: Supplementary figures and tables, experimental methods, procedures for synthesis, and full characterization data of compounds. See DOI: 10.1039/c6sc01279e involved in insulin resistance and revealed that anti-diabetic PPAR $\gamma$ ligands effectively block this phosphorylation. ${ }^{5}$ Based on these findings, it was suggested that the efficacy of anti-diabetic PPAR $\gamma$ ligands is attributed to their ability to inhibit the phosphorylation of PPAR $\gamma$. Moreover, it was recently elucidated that an extracellular signal-regulated kinase (ERK)/cyclin-dependent kinase 5 (Cdk5) axis regulates the diabetogenic actions of PPAR $\gamma$ through the phosphorylation of Ser273, ${ }^{6}$ and that Ser273-phosphorylated PPAR $\gamma$ is selectively recognized by thyroid hormone receptor-associated protein 3 (Thrap3) and regulates a diabetic gene set through these signaling pathways. ${ }^{7}$

Although glitazones have remarkable effects in the treatment of type II diabetes, their use has declined because of their serious adverse effects including weight gain, fluid retention, and congestive heart failure. ${ }^{\mathbf{8} 9}$ Glitazones both inhibit the phosphorylation of Ser273 and fully activate the expression of PPAR $\gamma$ target genes, which is referred to as classical transcriptional agonism. ${ }^{5}$ Full classical agonism has been suspected of causing serious side effects; thus, there have been consistent efforts to develop a selective PPAR $\gamma$ modulator (SPPAR $\gamma M$ ) that exhibits reduced classical agonism while retaining potent effects on insulin sensitization. ${ }^{\mathbf{1 0 - 1 2}}$ Because PPAR $\gamma$ has a large binding pocket and multiple interaction points with ligands, it is expected that PPAR $\gamma$ activities can be selectively regulated through the site-specific binding of ligands. ${ }^{13}$ The recent 
discovery of SR1664 as a representative SPPAR $\gamma \mathrm{M}$ helped demonstrate that the complete and selective modulation of PPAR $\gamma$ activities is possible via specific ligand binding. Unlike glitazones, SR1664, which inhibits the phosphorylation of Ser273 without altering the transcriptional activity of PPAR $\gamma$, exerts potent in vivo anti-diabetic effects without causing fluid retention and weight gain. ${ }^{14}$ In consideration of these results, there is no doubt that designing a selective inhibitor of PPAR $\gamma$ phosphorylation can be a powerful strategy for the development of a novel anti-diabetic agent targeting PPAR $\gamma$. However, the exact structural mechanism by which anti-diabetic PPAR $\gamma$ ligands block Ser273 phosphorylation has not yet been elucidated.

\section{Results and discussion}

\section{Alternate site binding of SR1664 blocks phosphorylation of PPAR $\gamma$ at Ser273}

To understand the exact molecular mechanism by which antidiabetic PPAR $\gamma$ ligands can inhibit Ser273 phosphorylation, we resolved the crystal structure of the PPAR $\gamma$ ligand-binding domain (LBD) complexed with SR1664 and the SRC1 coactivator peptide to a resolution of $2.20 \AA$ (Fig. 1a and S1a $\dagger$ ). As the molecular interaction between PPAR $\gamma$ and SR1664 was mainly associated with the inhibition of Ser273 phosphorylation, we might gain more insight into the mechanism by which antidiabetic PPAR $\gamma$ ligands block the phosphorylation of Ser273, by analyzing the co-crystal structures of the PPAR $\gamma$ LBD and SR1664. SR1664 had a completely different binding mode from full $\mathrm{PPAR} \gamma$ agonists such as rosiglitazone which binds at the canonical binding pocket of PPAR $\gamma$ via strong hydrogen bonding with helix 12 (Fig. 1b, blue). ${ }^{16}$ In contrast, SR1664 bound to an alternate site which is defined as the region near the entrance of the canonical binding pocket occupied by the second MRL-20 molecule when two molar equivalents of an MRL-20 ligand are bound to PPAR $\gamma$ (Fig. 1b, green). ${ }^{17}$

To determine the phosphorylation-inhibiting effect of SR1664 bound at the alternate site, we investigated using GW9662, a synthetic irreversible PPAR $\gamma$ inhibitor that covalently binds to cysteine 313 (Cys313 in PPAR $\gamma 2$; Cys285 in PPAR $\gamma 1$ ) on helix $3(\mathrm{H} 3){ }^{18}$ This covalent inhibitor completely blocks ligand engagement at the canonical binding pocket without fully inhibiting the alternate site binding of PPAR $\gamma$ ligands. ${ }^{17}$ In this study, GW9662 was used to block the canonical binding pocket to determine whether the alternate site binding of SR1664 affects coregulatory interactions. ${ }^{17}$ However, based on the X-ray co-crystal structure, we found that SR1664 exhibited a steric clash with the phenyl group of GW9662 (Fig. S2 $\dagger$ ), which can interfere in the binding event of SR1664 at the alternate site of PPAR $\gamma$ when this ligand engages PPAR $\gamma$ with the binding mode shown in the crystal structure.

Therefore, we designed and synthesized a smaller covalent inhibitor, SB1404 (1), by replacing the phenyl group with a methyl group (Fig. 1c). Compared to rosiglitazone or SR1664, SB1404 did not inhibit the Cdk5-mediated phosphorylation of $\operatorname{PPAR} \gamma$ at any concentration in vitro (Fig. 1d). Nevertheless, SB1404 completely blocked the inhibitory effect of rosiglitazone
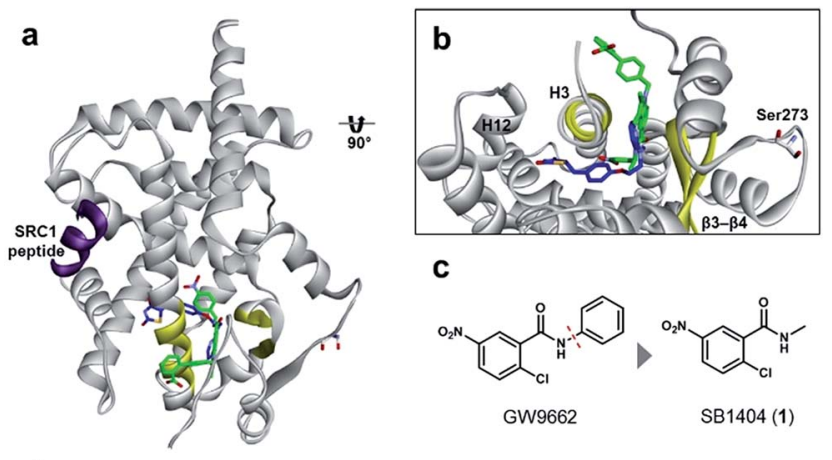

c

d
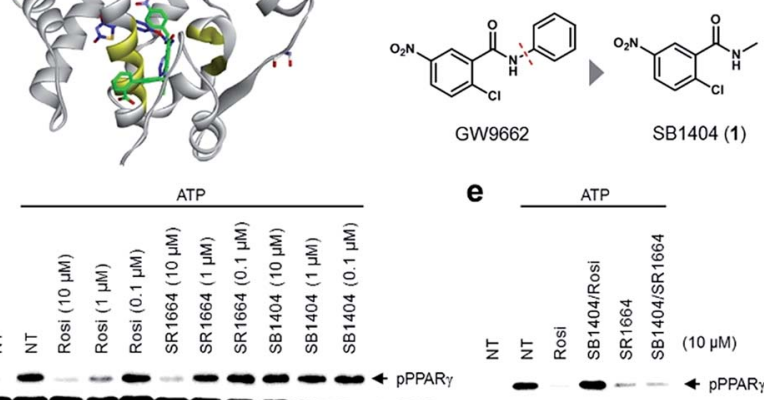

e

ATP

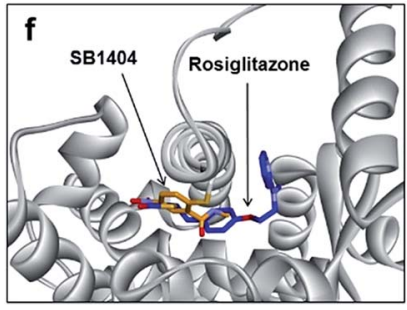

g

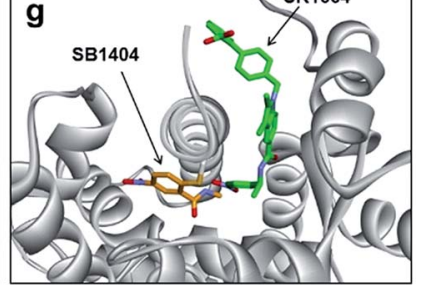

Fig. 1 Structural elucidation of the binding mode of SR1664 which blocks PPAR $\gamma$ phosphorylation at Ser273. ( $a, b)$ Alignment of the SR1664-PPAR $\gamma$ LBD (green) and rosiglitazone-PPAR $\gamma$ LBD (blue, PDB: 2PRG) X-ray co-crystal structures. (c) Design of a small PPAR $\gamma$ antagonist, SB1404. (d) In vitro Cdk5 assay of PPAR $\gamma$ in the presence of rosiglitazone, SR1664 or SB1404. (e) In vitro Cdk5 assay on PPAR $\gamma$ treated by rosiglitazone or SR1664 with or without SB1404. NT, no treatment; pPPAR $\gamma$, phosphorylated PPAR $\gamma$. (f) Alignment of the rosiglitazone-PPAR $\gamma$ LBD (blue, PDB: 2PRG) and SB1404-PPAR $\gamma$ LBD (orange) X-ray co-crystal structures. (g) Alignment of the SR1664PPAR $\gamma$ LBD (green) and SB1404-PPAR $\gamma$ LBD (orange) X-ray co-crystal structures.

on PPAR $\gamma$ phosphorylation, but it did not affect the inhibition of PPAR $\gamma$ phosphorylation by SR1664 (Fig. 1e).

Furthermore, on the basis of the X-ray crystal structure of the PPAR $\gamma$ LBD complexed with SB1404 and the SRC1 coactivator peptide (resolution, $2.80 \AA$ ), we confirmed that SB1404 covalently bound to Cys313 on $\mathrm{H} 3$ and completely blocked the binding of rosiglitazone at the canonical binding pocket of PPAR $\gamma$ (Fig. 1f and S3†े). Unlike GW9662, SB1404 exhibited no steric clash with SR1664; thus, SR1664 can bind to SB1404labeled PPAR $\gamma$ with the mode shown in the crystal structure (Fig. 1g), indicating that alternate site binding of SR1664 directly inhibits PPAR $\gamma$ phosphorylation. In fact, a different binding mode of SR1664 was recently reported, ${ }^{15}$ but it is not possible for SR1664 to bind to SB1404-labeled PPAR $\gamma$ through the reported binding mode (Fig. S4 $\dagger$ ). Although more studies are necessary to determine which conformation of ligand binding is the major one, we clearly determined the functional effect of the alternate site binding of SR1664 in terms of phosphorylation inhibition at Ser273 by Cdk 5 . 


\section{Site-specific binding of PPAR $\gamma$ ligands causes inhibition of the phosphorylation}

The common biological effect of both SR1664 and rosiglitazone is the specific inhibition of PPAR $\gamma$ phosphorylation at Ser273, which means that they should share a common characteristic. ${ }^{14}$ By analyzing the binding modes of SR1664 and rosiglitazone in $\mathrm{X}$-ray co-crystal structures, we found that both ligands occupied a specific hydrophobic region between helix $\mathrm{H} 3$ and the $\beta 3-\beta 4$ loop (Fig. 2a). Based on this structural insight, we hypothesized that the ligand binding at this specific binding site is responsible for the inhibition of PPAR $\gamma$ phosphorylation at Ser273. To test this hypothesis, we designed two covalent inhibitors that could selectively bind at the alternate site. Considering the structures of GW9662 and SB1404, it was found that a 2-chloro5-nitrobenzamide moiety can serve as an electrophile and covalently trap Cys313 regardless of the functional groups attached to the amide moiety (Fig. S3 $\uparrow$ ). Therefore, we rationally designed and synthesized SB1405 (2) and SB1406 (3) containing 2-(benzyloxy)phenyl and 3-(benzyloxy)phenyl groups, respectively, instead of the phenyl group in GW9662 (Fig. 2b and c).

On the basis of the GW9662-PPAR $\gamma$ co-crystal structure, we expected that the additional hydrophobic benzyl moiety would occupy the specific binding site of PPAR $\gamma$ between $\mathrm{H} 3$ and $\beta 3-$ $\beta 4$, and conducted in vitro Cdk5 assays using the synthesized covalent inhibitors. Only SB1405 inhibited the phosphorylation of PPAR $\gamma$ (Fig. 2d) without blocking the phosphorylation of the C-terminal fragment of the retinoblastoma protein $(\mathrm{Rb}$ peptide), a well-known Cdk5 substrate (Fig. $2 \mathrm{e}$ ). ${ }^{19}$ This indicated that SB1405 does not affect the fundamental kinase function of Cdk 5 but blocks the phosphorylation of PPAR $\gamma$ at Ser273 similar to the effects of rosiglitazone and SR1664. However, we did not observe this inhibitory activity in the case of SB1406, which is the structural isomer of SB1405. To explain this intriguing result, we resolved the crystal structures of the PPAR $\gamma$ LBD complexed either with SB1405 or SB1406 to resolutions of 2.75 or $2.95 \AA$ A, respectively. Similarly to SB1404, both compounds covalently bound to Cys313, but they displayed different binding modes (Fig. S5†). In particular, the benzyl group of SB1405 occupied the specific binding site of PPAR $\gamma$ (Fig. 2f), whereas the same moiety of SB1406 did not occupy this region (Fig. 2g). Therefore, these co-crystal structures clearly elucidated why only SB1405 inhibited PPAR $\gamma$ phosphorylation at Ser273, demonstrating that the occupation of the hydrophobic alternate site of PPAR $\gamma$ is essential for the inhibition of PPAR $\gamma$ phosphorylation.

When we aligned the co-crystal structures of the SB1404PPAR $\gamma$ LBD and SB1405-PPAR $\gamma$ LBD, we did not observe any considerable differences in their backbone conformations with

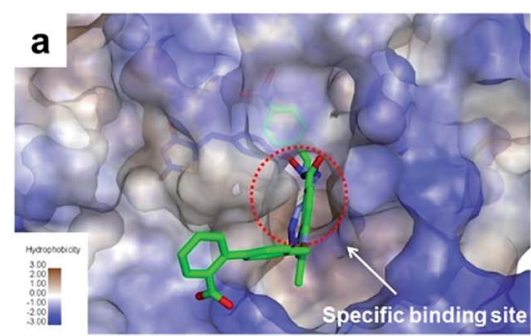

d

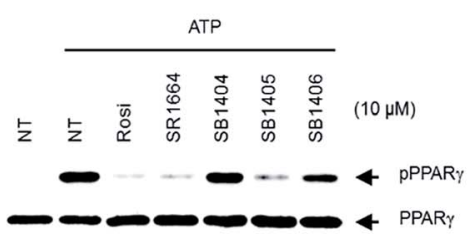

e

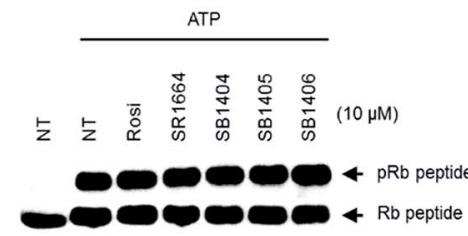

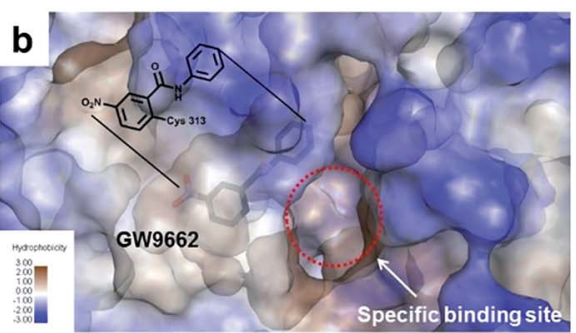
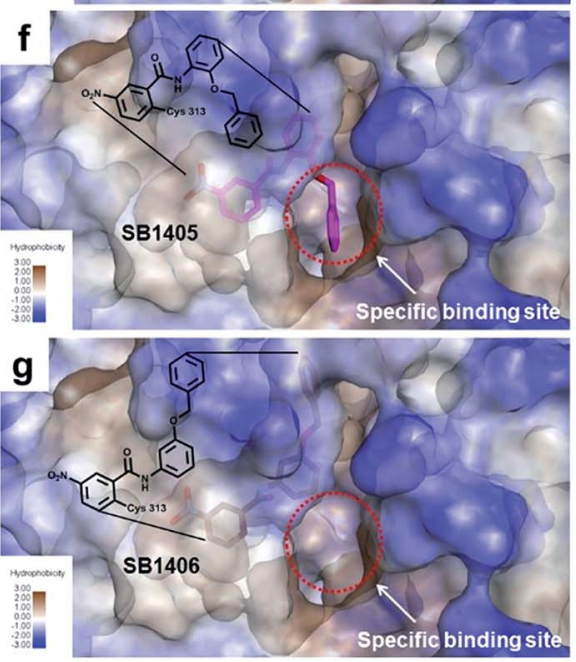

C

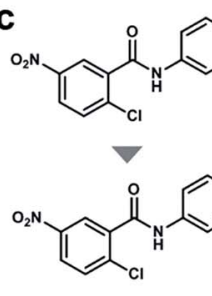

h
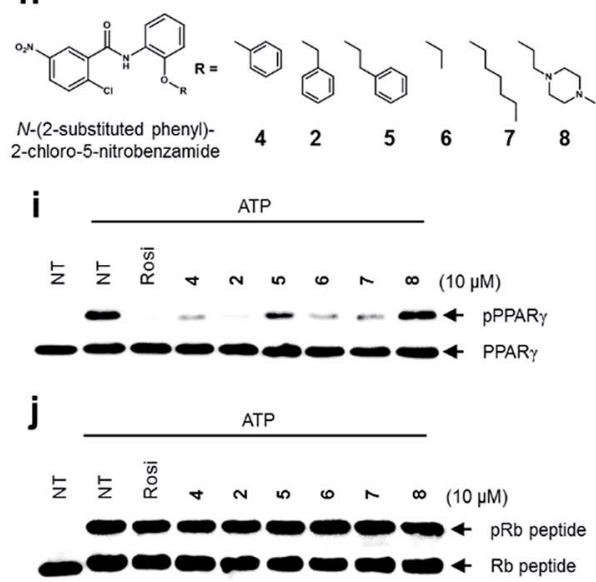

Fig. 2 Structure-based rational design of covalent inhibitors of PPAR $\gamma$ phosphorylation. (a) The proposed specific binding site in the SR1664PPAR $\gamma$ LBD X-ray co-crystal structures (green). (b) The specific binding site on the surface of the PPAR $\mathrm{LBD}$ complexed with GW9662 (black, PDB: 3B0R). (c) Structure-based design of the covalent inhibitors SB1405 and SB1406, which were expected to bind at the specific site. (d, e) In vitro Cdk5 assay of PPAR $\gamma$ or the Rb peptide on treatment with rosiglitazone, SR1664, or the covalent inhibitors. NT, no treatment; pPPAR $\gamma$, phosphorylated PPAR $\gamma$; pRb peptide, phosphorylated Rb peptide. (f, g) Binding modes of SB1405 (pink) and SB1406 (brown) confirmed by X-ray crystallography. (h) Chemical structures of $\mathrm{N}$-(2-substituted phenyl)-2-chloro-5-nitrobenzamides. (i, j) In vitro Cdk5 assay of PPAR $\gamma$ or the Rb peptide on treatment with rosiglitazone or $\mathrm{N}$-(2-substituted phenyl)-2-chloro-5-nitrobenzamides. 
a root-mean-square deviation (RMSD, C $\alpha$ ) of $0.36 \AA$, and there was no significant difference in the positioning of the residues around the specific binding site (Fig. S6†), which is consistent with previous crystallography study of PPAR $\gamma .^{20}$ Based on this structural information, we assumed that the inhibition of PPAR $\gamma$ phosphorylation is not an outcome of conformational changes but that it is probably caused by ligand-induced changes in the dynamic nature of the PPAR $\gamma$ LBD. To test this hypothesis, we performed a hydrogen/deuterium exchange (HDX) experiment with mass spectrometry. As shown in Fig. 3 and S7, $\uparrow$ SB1405, but not SB1404, significantly reduced the hydrogen/deuterium exchange rate in the $\beta$-sheet compared to the ligand-free PPAR $\gamma$ LBD. This ligand-induced reduction of the hydrogen/deuterium exchange rate at that site is an indication of change in the dynamic nature of $\beta$-sheet, and the reduced flexibility of this region probably results in the subsequent inhibition of Cdk5-mediated PPAR $\gamma$ phosphorylation at Ser273. This concept has been discussed in previous studies based on results that PPAR $\gamma$ phosphorylation inhibitors commonly stabilized $\mathrm{H} 3$ and the $\beta$-sheet region. ${ }^{\mathbf{5 , 1 4 , 1 7}}$ However, we clearly demonstrated this concept by comparing the HDXMS results of SB1404 and SB1405. Moreover, SB1405 did not cause any structural dynamic changes on the C-terminal indicating that SB1405 acts as a partial or non-agonist of PPAR $\gamma$, while anti-diabetic PPAR $\gamma$ ligands including rosiglitazone decrease hydrogen/deuterium exchange at the C-terminal as well as the $\beta$-sheet region of PPAR $\gamma$ in recent HDX studies. ${ }^{5}$

Based on the mechanistic and structural understanding of PPAR $\gamma$ phosphorylation, we selected $N$-(2-substituted phenyl)-2chloro-5-nitrobenzamide as a suitable molecular framework and synthesized a series of covalent inhibitors of PPAR $\gamma$ phosphorylation containing different $\mathrm{R}$ groups to effectively occupy this specific binding site (Fig. 2h). As shown in Fig. 2i, these inhibitors displayed good inhibitory activities toward Cdk5mediated in vitro PPAR $\gamma$ phosphorylation, excluding 8 which contains a hydrophilic piperazine moiety. In contrast, all these compounds exerted no inhibitory effect on the phosphorylation of the Rb peptide by Cdk5 (Fig. 2j). Taken together, the $\mathrm{N}$-(2-

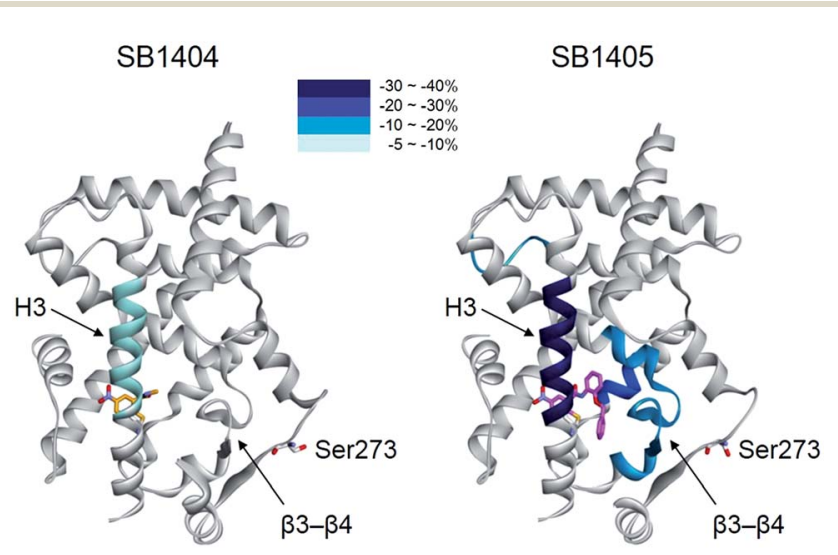

Fig. 3 Overlay of differential HDX data onto the structures of the PPAR $\gamma$ LBD bound with either SB1404 or SB1405. Percentage difference in HDX between the apo and ligand-bound receptor is coloured according to the key. substituted phenyl)-2-chloro-5-nitrobenz-amides possessing hydrophobic R substituents efficiently blocked Cdk5-mediated PPAR $\gamma$ phosphorylation at Ser273, and the occupation of $\mathrm{R}$ substituents at the hydrophobic region between $\mathrm{H} 3$ and $\beta 3-\beta 4$ of PPAR $\gamma$ appears to be sufficient for reducing the flexibility of this region, which inhibits the in vitro phosphorylation of PPAR $\gamma$ by Cdk5.

\section{Structure-guided optimization and evaluation of covalent inhibitors for in vivo analysis}

Among the $N$-(2-substituted phenyl)-2-chloro-5-nitrobenzamides, we selected SB1405 (2) for a further in vivo study because of its excellent inhibition potency in the in vitro Cdk5 assay (Fig. 2i) and the perfect occupancy of its benzyl moiety at the specific binding site (Fig. 2f). However, we were concerned that the in vivo evaluation of SB1405 might be limited due to its poor solubility. To solve this problem, we decided to modify the structure of SB1405 without changing the 2-chloro-5-nitrobenzamide moiety to preserve covalent anchoring with Cys313 of PPAR $\gamma$ and a hydrophobic benzyl moiety to specifically bind the alternate site for the phosphorylation inhibition. Thus, we introduced an additional moiety on the benzyl moiety of SB1405 to occupy the remaining empty hydrophobic space (brown) and the subsequent hydrophilic space (blue) (Fig. 4a). On the basis of the crystal structure of the SB1405-PPAR $\gamma$ LBD, we designed and synthesized SB1451 (9) and SB1453 (10) containing hydrophilic piperazine moieties attached to the additional benzene rings to improve solubility (Fig. 4b). After synthesizing SB1451 and SB1453, we examined their inhibitory activities against PPAR $\gamma$ phosphorylation at Ser273 in a concentration-dependent

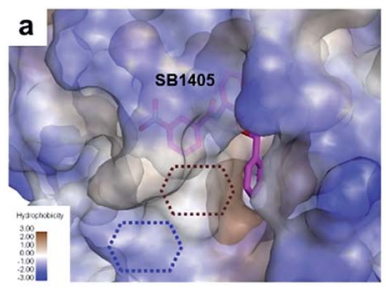

c
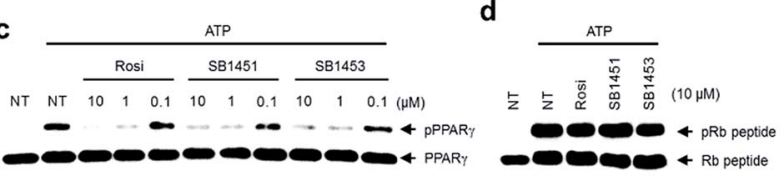

e

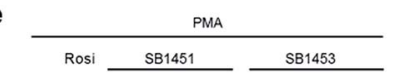

NT $\quad$ NT $110 \begin{array}{llllllllll}10 & 1 & 0.1 & 0.01 & 10 & 1 & 0.1 & 0.01 & (\mu M)\end{array}$
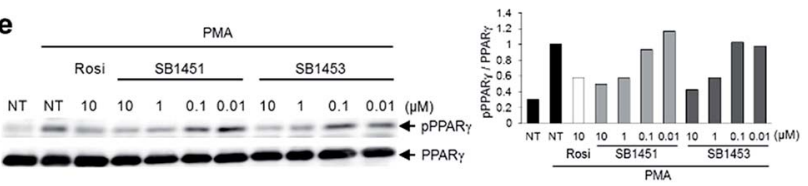

Fig. 4 Rational optimization of covalent PPAR $\gamma$ phosphorylation inhibitors for in vivo analysis. $(a, b)$ Structure-guided rational design of the covalent inhibitors SB1451 and SB1453 to improve the solubility of SB1405. (c, d) In vitro Cdk5 assay of PPAR $\gamma$ or the Rb peptide after treatment with rosiglitazone, SB1451, or SB1453. NT, no treatment; pPPAR $\gamma$, phosphorylated PPAR $\gamma$; pRb peptide, phosphorylated $\mathrm{Rb}$ peptide. (e) PMA-induced phosphorylation of PPAR $\gamma$ in HEK-293 cells expressing PPAR $\gamma$ after treatment with rosiglitazone, SB1451, or SB1453. 
manner and confirmed their excellent potency in the in vitro Cdk5 assay (Fig. 4c). This inhibitory potency of SB1451 and SB1453 regarding PPAR $\gamma$ phosphorylation was also conserved in the cellular system, which was similar to that of rosiglitazone (Fig. 4e). However, they did not inhibit the basic kinase function of Cdk5 as monitored by a Western blot analysis of Rb peptide phosphorylation in vitro (Fig. 4d).

\section{Covalent PPAR $\gamma$ phosphorylation inhibitors exert anti- diabetic effects in vivo without promoting adipogenesis}

To examine the agonistic activity in PPAR $\gamma$-mediated transcription, we conducted two cellular assays. First, using a luciferase reporter gene assay in HEK-293T cells expressing fulllength PPAR $\gamma$ with tandem PPAR response elements (PPRE), we confirmed that SB1451 and SB1453 had almost no classical transcriptional agonism compared with that of rosiglitazone (Fig. 5a). Secondly, we tested whether these covalent inhibitors stimulate adipogenesis by monitoring cellular lipid accumulation in 3T3-L1 cells via Oil Red O staining. ${ }^{21,22}$ As shown in Fig. 5b, rosiglitazone $(10 \mu \mathrm{M})$ fully stimulated adipocyte
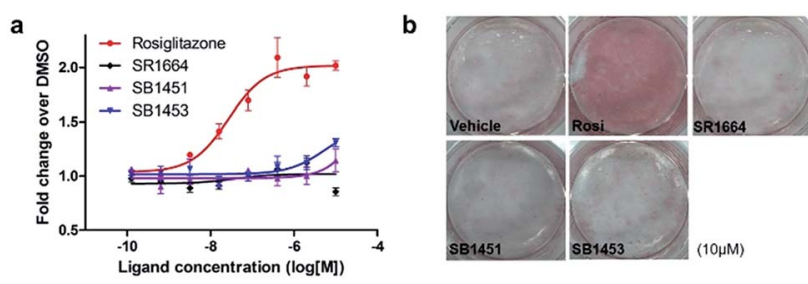

C

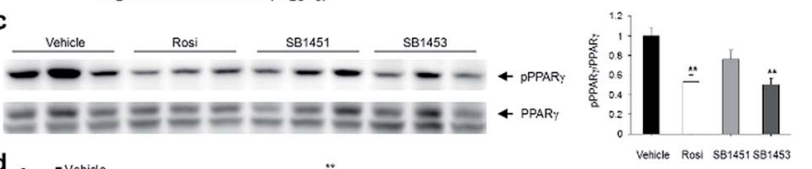

d 5 - vehide

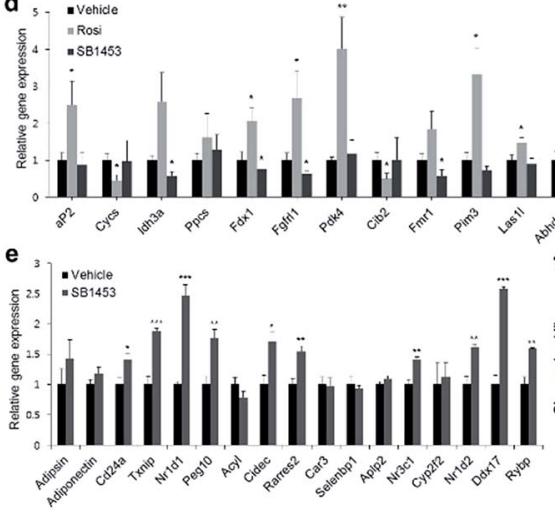

Fig. 5 Anti-diabetic activity of SB1453 in DIO mice without promoting adipogenesis. (a) Transcriptional activity of a PPAR-derived reporter gene in HEK-293T cells expressing full-length PPAR $\gamma$ after $24 \mathrm{~h}$ of treatment with rosiglitazone, SR1664, SB1451, or SB1453 ( $n=3$ ). (b) Oil Red $O$ staining of accumulated lipids in 3T3-L1 cells treated with rosiglitazone, SR1664, SB1451, or SB1453. (c) Phosphorylation of PPAR $\gamma$ in the WAT of DIO mice after 7 days of treatment with vehicle, rosiglitazone, SB1451, or SB1453 (10 mg per kg per day). (d) Expression of the agonist gene set in WAT. (e) Expression of the gene set regulated by PPAR $\gamma$ phosphorylation in WAT. (f) Glucose-tolerance test in DIO mice treated with vehicle, rosiglitazone, or SB1453 (7 days, $10 \mathrm{mg}$ per kg per day) $(n=6)$. Error bars denote SEM. $* P<0.05, * * P<0.01$, $* * * P<0.001$. differentiation, whereas SB1451 or SB1453 $(10 \mu \mathrm{M})$ did not trigger adipogenesis in 3T3-L1 cells, which confirms the lack of transactivation associated with these inhibitors. Although we observed a slight increase in the PPAR $\gamma$ transcriptional activity upon treatment with SB1453 at $10 \mu \mathrm{M}$ in the luciferase assay, this concentration was insufficient to stimulate the differentiation of pre-adipocytes into mature adipocytes.

We evaluated the anti-diabetic activity of SB1451 and SB1453 in animal models using DIO mice that are insulin-resistant with an increased level of phosphorylated PPAR $\gamma$ at Ser273. ${ }^{5}$ All animal experiments were performed according to procedures approved by Ulsan National Institute of Science and Technology's Institutional Animal Care and Use Committee. As shown in Fig. 5c, SB1453 effectively decreased the phosphorylation of PPAR $\gamma$ at Ser273 in the white adipose tissue (WAT) of the DIO mice similarly to the effect of rosiglitazone. SB1451 was less potent in both the in vivo reduction of PPAR $\gamma$ phosphorylation and the resulting anti-diabetic activity than SB1453. Previous studies clearly demonstrated that SR1664, a selective inhibitor of PPAR $\gamma$ phosphorylation without classical agonism, exerts in vivo anti-diabetic effects and causes changes in the expression of diabetic genes that were dysregulated as a result of PPAR $\gamma$ phosphorylation in obese animals. ${ }^{12,14}$ Similarly to SR1664, SB1453 altered the expression of 10 out of 17 affected genes (Fig. 5e). Furthermore, we did not observe any SB1453-induced stimulation of the "agonist" gene set in the white adipose tissue of the DIO mice as defined in a previous report ${ }^{14}$ (Fig. $5 \mathrm{~d}$ ). The glucose tolerance in the DIO mice was improved with the treatments of SB1453 at $10 \mathrm{mg}$ per $\mathrm{kg}$ per day for 7 days, although this effect was moderate compared with that of rosiglitazone (Fig. 5f). These results indicated that SB1453 has antidiabetic actions and preferentially regulates genes sensitive to PPAR $\gamma$ phosphorylation.

We also investigated several adverse effects, including fluid retention and cardiac hypertrophy, which have been observed following treatment with glitazones. ${ }^{\mathbf{8} 23}$ As shown in Fig. S8a, $\dagger$ the treatment of rosiglitazone caused hemodilution, whereas the treatment of SB1453 had no detectable changes compared with vehicle. Furthermore, the expressions of natriuretic peptide type B (BNP), the marker gene of heart failure, or myosin heavy chain $\beta$ ( $\beta$-MHC), the marker gene of hypertrophy, were significantly increased in only rosiglitazone-treated mice without changes in the heart weight (Fig. S8b and c $\dagger$ ). These results strongly suggest that SB1453 does not induce the adverse effects associated with the in vivo treatment of glitazones.

\section{Covalent inhibitor selectively binds to PPAR $\gamma$}

Lastly, we confirmed the target selectivity of SB1453 using a target identification probe. Prior to the probe design, we resolved the crystal structure of the PPAR $\gamma$ LBD complexed with SB1453 and the SRC1 coactivator peptide (resolution, 2.30 A) to gain more information about its binding mode (Fig. 6a). As expected, SB1453 covalently bound to Cys313 on H3 and occupied the hydrophobic region between $\mathrm{H} 3$ and $\beta 3-\beta 4$. In addition, its piperazine moiety was positioned at the entrance of the PPAR $\gamma$ binding pocket. Based on this structural information, we 


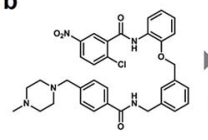

SB1453 (10)

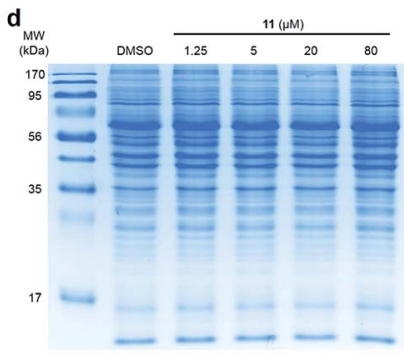

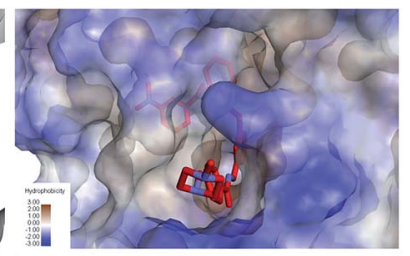
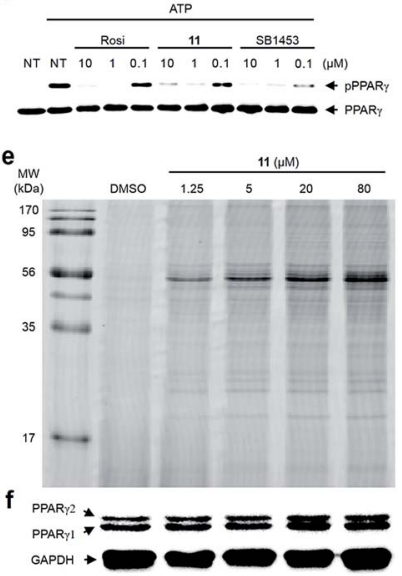

Fig. 6 (a) X-ray co-crystal structure of the SB1453-PPAR $Y$ LBD reveals the exposure of the piperidine moiety at the entrance of the PPAR $\gamma$ binding pocket. (b) Structure-guided rational design of the target identification probe 11 to demonstrate the target selectivity. (c) In vitro Cdk5 assay of PPAR $\gamma$ on treatment with rosiglitazone, 11, or SB1453 in a dose-dependent manner. (d, e) Coomassie staining and fluorescence scanning patterns of SDS-PAGE of lysates from differentiated 3T3-L1 adipose cells treated with probe 11 in various concentrations. (f) Western blot against the same gel in (e) to confirm PPAR $\gamma$ as the target proteins labeled by 11 .

designed the target identification probe $\mathbf{1 1}$ containing a terminal acetylene group on the piperazine moiety to enable the fluorescence labeling of target proteins via a bioorthogonal click reaction (Fig. 6b). Despite the structural change, probe $\mathbf{1 1}$ retained its phosphorylation inhibitory effect, indicating that this probe probably covalent bonded with Cys 313 on the $\mathrm{H} 3$ of PPAR $\gamma$ (Fig. 6c).

First, we confirmed the PPAR subtype selectivity of our covalent inhibitor. Probe $\mathbf{1 1}$ was incubated with the proteome of HEK-293T cells expressing either murine PPAR $\alpha$, PPAR $\delta$, or $\operatorname{PPAR} \gamma$, followed by a copper-catalyzed click reaction with an azide-containing Cy5 to visualize the proteins complexed with probe 11. The resulting proteome was separated by gel electrophoresis, and target proteins were visualized via fluorescence scanning. Predominantly, the fluorescence-labeled protein was only detected in the PPAR $\gamma$-transfected cells, indicating that probe 11 efficiently binds to PPAR $\gamma$, and not to PPAR $\alpha$ or PPAR $\delta$ (Fig. S9†). Then, we performed the same experiment with differentiated 3T3-L1 adipose cells. Interestingly, the predominant bands appeared on the fluorescent gel in a dose-dependent manner without any difference in protein expression pattern (Fig. 6d and e). These labeled proteins were identified as PPAR $\gamma 1(53 \mathrm{kDa})$ and PPAR $\gamma 2(57 \mathrm{kDa})$ by Western blot analysis (Fig. 6f). In fact, they are two isoforms of PPAR $\gamma$ and have a common LBD to which SB1453 binds. This result demonstrated that our covalent inhibitor SB1453 selectively binds to target protein and might be free from general concerns regarding the nonspecific binding of irreversible covalent inhibitors. ${ }^{24}$

\section{Conclusions}

In this study, we identified that an alternate site binding of SR1664 effectively blocks the phosphorylation of PPAR $\gamma$ at Ser273. By comparing this binding mode with that of the conventional PPAR $\gamma$ ligand rosiglitazone, we found that ligand binding at a specific binding site, the hydrophobic region between $\mathrm{H} 3$ and $\beta 3-\beta 4$, is closely related to the inhibition of PPAR $\gamma$ phosphorylation at Ser273. To determine the functional effect of PPAR $\gamma$ ligand binding at the specific site, we rationally designed and synthesized irreversible covalent inhibitors as chemical tools. By analysing the data from biochemical assays, X-ray crystallography, and HDX mass spectrometry with these covalent inhibitors, we found that occupation of the specific binding site in PPAR $\gamma$ by small-molecule ligands causes the change in the dynamic nature of $\beta$-sheet and directly correlates with the inhibitory effects on the phosphorylation of PPAR $\gamma$ at Ser273. This structural insight led us to rationally design the improved covalent inhibitors SB1451 and SB1453, which effectively inhibit the phosphorylation of PPAR $\gamma$ at Ser273 in vitro and in adipose cells. We also demonstrated that SB1453 exerts potent anti-diabetic effects in DIO mice by blocking PPAR $\gamma$ phosphorylation at Ser273 in the white adipose tissue without several of the adverse effects associated with glitazones. Lastly, through fluorescence-based visualization of the target proteins complexed with the covalent probe $\mathbf{1 1}$ containing a bioorthogonal functional handle, we confirmed that our potent anti-diabetic agent SB1453 selectively binds to PPAR $\gamma$. This study provides a useful guideline for the structure-based rational design of SPPAR $\gamma$ Ms that effectively inhibit the phosphorylation of Ser273 for the development of anti-diabetic PPAR $\gamma$ ligands. It can be expected that new classes of PPAR $\gamma$ targeting anti-diabetic drugs will be developed in accordance with this guideline.

\section{Acknowledgements}

This work was supported by the Creative Research Initiative Grant (2014R1A3A2030423), the Bio \& Medical Technology Development Program (2012M3A9C4048780), the Innovative Drug Research Center for Metabolic and Inflammation Disease (2007-0056817 to SWS) and the Global Research Lab Program (2012K1A1A2045441 to KJL) through the National Research Foundation of Korea (NRF) funded by the Korean Government (Ministry of Science, ICT \& Future Planning), and the Korean Health Technology R\&D Project funded by the Ministry of Health \& Welfare, Republic of Korea (HI14C2518 to JHC). We thank the beamline staff for assistance during X-ray diffraction experiments at Pohang Light Source (beamlines BL-5C and BL7A) and Photon Factory (beamlines BL-1A, BL-5A and NW12A). H. B., J. Y. J., H. K. and A. J. are grateful for their predoctoral fellowship awarded by the BK21 Plus Program. 


\section{Notes and references}

1 A. R. Saltiel and C. R. Kahn, Nature, 2001, 414, 799-806.

2 J. M. Lehmann, L. B. Moore, T. A. Smith-Oliver, W. O. Wilkison, T. M. Willson and S. A. Kliewer, J. Biol. Chem., 1995, 270, 12953-12956.

3 M. Lehrke and M. A. Lazar, Cell, 2005, 123, 993-999.

4 R. E. Soccio, E. R. Chen and M. A. Lazar, Cell Metab., 2014, 20, 573-591.

5 J. H. Choi, A. S. Banks, J. L. Estall, S. Kajimura, P. Boström, D. Laznik, J. L. Ruas, M. J. Chalmers, T. M. Kamenecka, M. Blüher, P. R. Griffin and B. M. Spiegelman, Nature, 2010, 466, 451-456.

6 A. S. Banks, F. E. McAllister, J. P. Comporez, P. J. Zushin, M. J. Jurczak, D. Laznik-Bogoslavski, G. I. Shulman, S. P. Gygi and B. M. Spiegelman, Nature, 2015, 517, 391-395.

7 J. H. Choi, S. S. Choi, E. S. Kim, M. P. Jedrychowski, Y. R. Yang, H. J. Jang, P. G. Suh, A. S. Banks, S. P. Gygi and B. M. Spiegelman, Dev. Gene. Evol., 2014, 28, 2361-2369.

8 R. W. Nesto, D. Bell, R. O. Bonow, V. Fonseca, S. M. Grundy, E. S. Horton, M. Le Winter, D. Porte, C. F. Semenkovich, S. Smith, L. H. Young and R. Kahn, Diabetes Care, 2004, 27, 256-263.

9 M. Ahmadian, J. M. Suh, N. Hah, C. Liddle, A. R. Atkins, M. Downes and R. M. Evans, Nat. Med., 2013, 19, 557-566.

10 S. M. Rangwala and M. A. Lazar, Sci. Signaling, 2002, pe9.

11 J. J. Acton 3rd, R. M. Black, A. B. Jones, D. E. Moller, L. Colwell, T. W. Doebber, K. L. Macnaul, J. Berger and H. B. Wood, Bioorg. Med. Chem. Lett., 2005, 15, 357-362.

12 S. S. Choi, E. S. Kim, M. Koh, S. J. Lee, D. Lim, Y. R. Yang, H. J. Jang, K. A. Seo, S. H. Min, I. H. Lee, S. B. Park, P. G. Suh and J. H. Choi, J. Biol. Chem., 2014, 289, 2661826629.

13 L. S. Higgins and A. M. Depaoli, Am. J. Clin. Nutr., 2010, 91, 267S-272S.

14 J. H. Choi, A. S. Banks, T. M. Kamenecka, S. A. Busby, M. J. Chalmers, N. Kumar, D. S. Kuruvilla, Y. Shin, Y. He,
J. B. Bruning, D. P. Marciano, M. D. Cameron, D. Laznik, M. J. Jurczak, S. C. Schürer, D. Vidović, G. I. Shulman, B. M. Spieglman and P. R. Griffin, Nature, 2011, 477, 477481.

15 D. P. Marciano, D. S. Kuruvilla, S. V. Boregowda, A. Asteian, T. S. Hughes, R. Garcia-Ordonez, C. A. Corzo, T. M. Khan, S. J. Novick, H. Park, D. J. Kojetin, D. G. Phinney, J. B. Bruning, T. M. Kamenecka and P. R. Griffin, Nat. Commun., 2015, 6, 7443.

16 R. T. Nolte, G. B. Wisely, S. Westin, J. E. Cobb, M. H. Lambert, R. Kurokawa, M. G. Rosenfeld, T. M. Willson, C. K. Glass and M. V. Milburn, Nature, 1988, 395, 137-143.

17 T. S. Hughes, P. K. Giri, I. M. de Vera, D. P. Marciano, D. S. Kuruvilla, Y. Shin, A. L. Blayo, T. M. Kamenecka, T. P. Burris, P. R. Griffin and D. J. Kojetin, Nat. Commun., $2014,5,3571$.

18 L. M. Leesnitzer, D. J. Park, R. K. Bledsoe, J. E. Cobb, J. L. Collins, T. G. Consler, R. G. Davis, E. A. Hull-Ryde, J. M. Lenhard, L. Patel, K. D. Plunket, J. L. Shenk, J. B. Stimmel, C. Therapontos, T. M. Willson and S. G. Blanchard, Biochemistry, 2002, 41, 6640-6650.

19 X. Grana, A. de Luca, N. Sang, Y. Fu, P. P. Claudio, J. Rosenblatt, D. O. Morgan and A. Giordano, Proc. Natl. Acad. Sci. U. S. A., 1994, 91, 3834-3838.

20 J. B. Bruning, M. J. Chalmers, S. Prasad, S. A. Busby, T. M. Kamenecka, Y. He, K. W. Nettles and P. R. Griffin, Structure, 2007, 15, 1258-1271.

21 P. Tontonoz, E. Hu and B. M. Spiegelman, Cell, 1994, 79, 1147-1156.

22 A. Chawla, E. J. Schwarz, D. D. Dimaculangan and M. A. Lazar, Endocrinology, 1994, 135, 798-800.

23 R. D. Hannan, A. Jenkins, A. K. Jenkins and Y. Brandenburger, Clin. Exp. Pharmacol. Physiol., 2003, 30, 517-527.

24 J. Singh, R. C. Petter, T. A. Baillie and A. Whitty, Nat. Rev. Drug Discovery, 2011, 10, 307-317. 